\title{
Science globale et interdisciplinarité : quand contagion des concepts rime avec confusion
}

On reproche souvent aux politiques de manier la langue de bois en mobilisant toute une série de termes qui, mis ensemble, finissent par donner l'impression d'un discours construit mais qui noient en réalité l'auditeur dans une logorrhée destinée à dissimuler parfois une absence d'idées. Ces travers bien connus du monde politique ont été dénoncés avec ironie par le comédien Franck Lepage. Dans l'une de ses conférences gesticulées ${ }^{1}$, il va même jusqu'à utiliser une série de cartes sur lesquelles des « concepts opérationnels » sont inscrits et il construit un discours en enchaînant ces mots les uns à la suite des autres, selon l'ordre que les cartes battues auront désigné : stupéfiant !

Cette pratique n'est cependant pas l'apanage des seuls politiques et on peut se demander aujourd'hui si l'essor d'une science globalisée où la communication scientifique entre disciplines passe par le biais de concepts flous, ne risque pas, in fine, de conduire à des dérives similaires. Quels sont les enjeux pour l'interdisciplinarité et comment éviter que le discours scientifique ne se limite à une simple rhétorique bien huilée ? C'est à ce type de questions que la revue NSS se trouve confrontée dans son activité éditoriale et pour lesquelles il nous a semblé utile d'offrir quelques pistes de réflexion.

Le discours scientifique dans le domaine de l'environnement n'échappe pas à la standardisation, mouvement qui tend à s'accélérer et conduit à la fois à un usage très intensif des concepts et à leur obsolescence rapide. La contagion des mots n'est pas un phénomène nouveau (rappelons-nous de l'essor des notions de développement durable, de biodiversité et de gouvernance dans les années 1990), elle se manifeste désormais au travers de concepts-valises qui sont appropriés d'autant plus facilement par ceux qui les utilisent qu'ils ont un caractère polysémique. Cette polysémie est tout autant source de richesse conceptuelle que de flou dans leur usage. Identifiés par ceux qui les utilisent dans un domaine spécifique, ils perdent de leur rigueur dans un discours

1 Voirhttp://www.youtube.com/watch?v=KGgkkN5nfos. global. L'adaptation (ou la gestion adaptative), les services écosystémiques, la transition (écologique ou énergétique), l'anthropocène, le bien commun, la vulnérabilité, la résilience, l'économie circulaire, sont autant d'exemples de ce que le discours scientifique véhicule aujourd'hui et dont s'emparent les politiques, les ONG et les citoyens. Loin de contester l'intérêt de chacun de ces concepts, pris isolément dans un contexte précis, ce qui nous paraît troublant est l'émergence d'un discours qui agrège tous ces termes ou les substitue l'un à l'autre. Contagion rime de plus en plus avec confusion.

Pourtant, l'usage de ces termes s'impose car ils opèrent comme autant de mots-clés qui peuvent aider à décrocher un contrat de recherche ou encore à attirer l'attention dans la soumission d'un résumé pour un colloque.

Cette situation pose un certain nombre d'enjeux pour l'interdisciplinarité. Celle-ci se construit souvent autour $\mathrm{d}$ 'objets communs et ces concepts peuvent être le moyen $\mathrm{d}^{\prime}$ initier un travail interdisciplinaire dès lors qu'ils permettent de réunir les membres d'un collectif autour de problématiques partagées. Néanmoins, leur usage peut aussi avoir des effets contre-productifs. Deux exemples.

Dans un article paru en 2010, R.B. Norgaard ${ }^{2}$ revient sur l'engouement suscité par la notion de « services écosystémiques », notamment au sein du groupe international d'experts impliqué au début des années 2000 dans le cadre du Millennium Ecosystem Assessment. Il rappelle qu'à l'origine, cette notion n'était qu'une métaphore visant à sensibiliser autour des enjeux de biodiversité et explique qu'un des effets pervers de cet engouement a été une simplification abusive du fonctionnement des écosystèmes vus comme un stock de capital naturel dispensant un flux de services écosystémiques. Or, comme le montre Norgaard, cette vision des choses est tout à fait compatible avec les approches économiques fondées sur

\footnotetext{
2 Norgaard, R.B., 2010. Ecosystem services: From eyeopening metaphor to complexity blinder, Ecological Economics, $69,6,1219-1227$.
} 
l'équilibre partiel, ce qui explique l'intérêt conjoint des économistes comme des écologues pour son usage. Cependant, ni l'économie ni les écosystèmes ne peuvent se résumer à un modèle fondé sur des stocks et des flux. Il s'agit d'une simplification qui occulte en réalité la complexité des phénomènes. Mais les publications scientifiques s'enchaînent sur le sujet, des groupes de travail internationaux se mettent en place, de nouvelles communautés scientifiques s'identifient autour de ces quelques mots... Cet exemple illustre tout à la fois l'intérêt (rapprochement disciplinaire) et les limites (simplification abusive) de tels concepts.

Citons également le succès de "résilience ", terme venu de la métallurgie, popularisé en France par B. Cyrulnik pour décrire le processus psychologique qui consiste pour un individu à résister à un traumatisme et à se reconstruire. Cette notion a été mobilisée dès les années 1970 avec celle de complex adaptive systems par des économistes, comme F. von Hayek, qui s'en est servi à l'appui des théories les plus néolibérales, mais aussi, dans une perspective très différente, par des écologues, comme C.S. Holling, pour exprimer la capacité des écosystèmes à évoluer afin de maintenir leurs fonctionnalités. Elle est reprise dans la théorie de la Panarchie et adoptée comme porte-drapeau d'une communauté scientifique internationale, surtout représentée aux États-Unis et en Europe du Nord, le réseau Resilience Alliance et sa revue Ecology and Society. Ce réseau organise des conférences scientifiques triennales, dont la troisième, Resilience and Development: Mobilizing for Transformation ${ }^{3}$, s'est tenue en mai de cette année à Montpellier (après Stockholm et Tempe, Arizona) : près de 1000 participants, scientifiques et non-scientifiques (décideurs, associatifs, etc.), des innovations réelles comme une demi-douzaine d'ateliers préparatoires ${ }^{4}$, une première journée off site sur 17 lieux, en ville comme à la campagne, ainsi que des événements musicaux et artistiques pendant toute la durée du colloque... Les 700 communications retenues, sur 1200 proposées, ont donné lieu à 165 sessions parallèles qui ont porté sur les sujets les plus divers, conceptuels comme sur la notion de socioécosystème, ou méthodologiques comme sur la métrologie de la résilience. Mais beaucoup d'autres contributions débordaient du cadre conceptuel $\mathrm{du}$ réseau. La notion de résilience envahit ainsi des domaines empiriques comme l'utilisation de ressources naturelles renouvelables, les systèmes urbains, le changement climatique et ses effets. Elle se substitue alors à d'autres termes devenus obsolètes: la durabilité (la « ville durable » devient « résilience urbaine »), l'adaptation (au changement climatique), etc. Or, le développement durable est un projet politique et normatif beaucoup plus large, l'adaptation est une forme de renoncement à la possibilité de politiques de prévention et mitigation. Utiliser " résilience » à leur place occulte ces enjeux.

Il s'agit d'un double renoncement pour la "résilience »: sortie des débats qui lui donnent sens à l'occasion de conférences scientifiques comme celle de Montpellier, elle perd de son potentiel analytique originel et, ignorant la portée politique et intellectuelle des termes qu'elle remplace, elle appauvrit le débat.

On pourrait poursuivre avec tous les autres concepts que nous avons cités. Mais ces effets de mode et de polarisation éphémère, qui caractérisent le fonctionnement actuel du monde scientifique, ne se font-ils pas aux dépens de l'approfondissement théorique... qui est la base du métier de chercheur ? Et, au-delà, de l'efficacité de l'action collective?

Ainsi, l'utilisation de ces termes dans le discours dominant, puis dans celui des politiques publiques et des conventions internationales, assise sur de telles confusions, contribue largement au glissement de sens et à la perte de la profondeur et de la rugosité de certaines expressions, au profit d'autres plus lisses et plus convenables, quoiqu'éphémères. Des concepts, ainsi vidés de leur sens, deviennent des instruments de communication au service d'actions annoncées avec éclat, mais sans suite. L'abus qui est fait d'indicateurs ou de guides «méthodologiques» ou de «bonnes pratiques» pour combler cette ambiguité conceptuelle est symptomatique d'une aggravation, plutôt que d'une réduction, de ce décalage entre les mots et l'heuristique qui leur est liée.

C'est dans la vocation de NSS d'ouvrir un espace de réflexions et de débats sur l'apport de ces concepts et les dérives issues de leur usage immodéré. Il ne s'agit pas pour nous de dénier l'importance de la production conceptuelle dans la dynamique des sciences de l'environnement, et dans celle des pratiques collectives, mais d'en appeler à plus de réflexivité sur leur contenu et leur association.

Olivier Petit, Bernard Hubert et Jacques Theys

\footnotetext{
3 Voir dans ce même numéro, le texte de Dominique Hervé et Mylène Rivière qui rend compte de cette conférence : Hervé, D., Rivière, M., 2014. Résilience, adaptation, changement : l'interdisciplinarité questionnée, NSS, 22, 3.

4 Dont l'un au cours du colloque « Interdisciplinarités entre Natures et Sociétés » organisé par NSS à Cerisy-la-Salle en octobre 2013...
} 